\title{
Apport de la modélisation de la pluie en débit pour la connaissance de la ressource en eau et la prédétermination des crues
}

\author{
The contribution of rainfall-flow modelling to the understanding \\ of water resources and flood prediction
}

\author{
par J. Lavabre*, P. Arnaud*, J.M. Masson**, N. Folton* \\ * Cemagref Aix-en-Provence \\ ** Groupe hydrologie de l'UMR 5569, Université de Montpellier
}

The present availability of powerful calculation means and the geographical data bases allow to get beyond the simple statistics in order to solve the hydrological problems in assessing the water resource and in predetermining the floods. In conjunction with rain generators, some modelizations of the rain into discharge are able to generate continuous long time series of discharge and flood hydrograms. These time series are then available to take up different problematics : comparison resource-need, water reservoir management rules, design flood... The use of simple models allows to catch sight of real opportumities of regionalisation and integration into some geographical information systems as well as simulation opportumities of the impacts linked to anthropical activities.

\section{INTRODUCTION}

La loi d'orientation, d'aménagement et de développement du territoire affiche, comme il se doit, un souci de respect du territoire. Elle définit en outre un schéma des espaces naturels et des risques pour assurer la préservation et la valorisation des ressources patrimoniales, avec un objectif de réduction des risques. L'Eau est bien sûr concernée dans cette démarche : eau milieu vivant, eau vecteur, eau ressource renouvelable mais aléatoire, eau et ses pénuries, eau et ses excès...

Bien gérer la problématique de l'eau implique une connaissance aussi parfaite que possible de la disponibilité de la ressource, de sa variabilité, des phénomènes extrêmes.... et autant que faire ce peut proposer des scénarios prospectifs pour l'analyse du risque et la gestion des crises, dans un avenir où les effets anthropiques sont mal maîtrisés (changement climatique, occupation de l'espace ...).

Et on peut faire le constat que, malgré le développement des puissances de calcul, la disponibilité d'outils tels que les modèles numériques de terrain, l'imagerie satellitale, la technicité hydrologique n'évolue que très lentement et l'ingénierie reste dépourvue d'outils hydrologiques susceptibles d'aborder l'ensemble des problèmes posés.

Depuis quelques années, le Cemagref - notamment les unités de recherche en hydrologie d'Antony et d'Aix-en-Provence - s'est investi dans des recherches sur la modélisation de la pluie en débit afin de mettre à disposition de la com- munauté hydrologique des outils performants d'utilisation facile. Nous reportons ici les résultats de ces recherches pour la connaissance de la ressource en eau et des écoulements de crue des bassins versants.

\section{II — LA MODÉLISATION : INTÉRÊT POUR LA PRÉDÉTERMINATION DES DÉBITS}

Quel que soit leur degré de sophistication, les méthodes hydrologiques ont au moins un point en commun : elles ne peuvent se passer de l'information des réseaux de mesure, et leurs performances sont étroitement liées à la disponibilité des chroniques d'informations (dans le temps et dans l'espace).

La modélisation des phénomènes reste empirique ( $\mathrm{y}$ compris pour les modèles dits physiques) et fait essentiellement appel à des techniques numériques : statistiques, modèles stochastiques, modèles déterministes... L'ensemble de ces techniques reste tributaire de l'information disponible, notamment les statistiques simples effectuées sur une variable unique (la pluie maximale annuelle de durée une heure, le débit maximal annuel de pointe de crue...) qui sont, par construction, fortement dépendantes de l'échantillon étudié. L'exemple de la chronique des débits de crue du bassin versant du Bevinco à Lancone (Haute-Corse) est assez éloquent : les deux événements observés en 1993 et 1994 se distinguent nettement de la chronique 1974-1992 (figure 1). Une loi de probabilité à décroissance exponentielle représentait correctement les débits de la chronique 1972-1992 ; le 


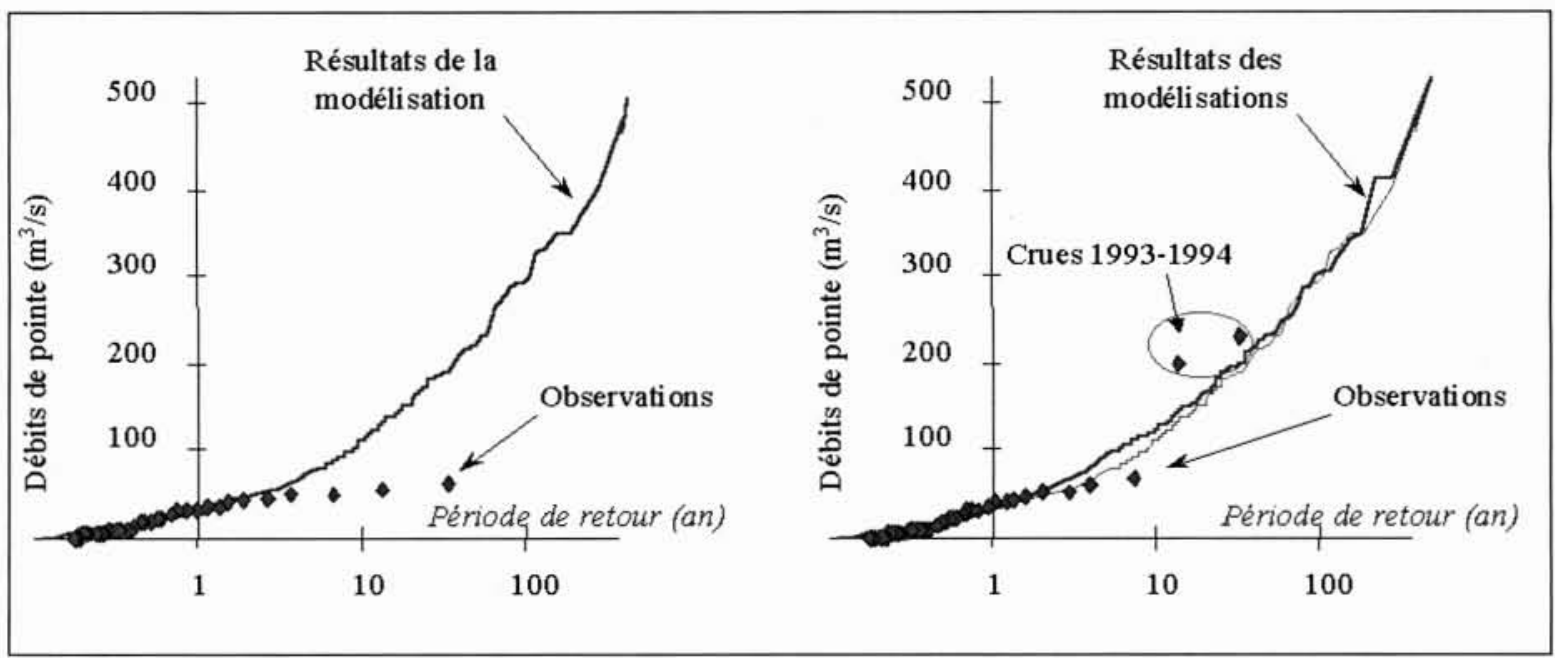

1. Bassin versant du Bevinco à Lancone (Haute-Corse). Distribution de fréquence des débits maxima annuels (graphique gauche : période 1972-1992 ; graphique droit : 1972-1994) et résultats des modélisations.

débit de crue de fréquence décennale était estimé autour de $60 \mathrm{~m}^{3} / \mathrm{s}$ (figure 1, graphique gauche). Il apparaît bien plus délicat d'avancer une estimation de ce quantile à l'examen de l'échantillon 1972-1994 (figure 1, graphique de droite).

Il semblerait que des modèles à peine plus compliqués puissent se départir des problèmes liés à l'échantillonnage. Ainsi, les résultats obtenus par une modélisation de la pluie en débit associée à un générateur stochastique de pluies horaires sont à l'évidence bien moins sensibles à l'échantillonnage : les distributions de fréquence calculées sur les chroniques 1974-1992 et 1974-1994 sont tout à fait comparables (figure 1, graphique de droite).

La méthode qui peut être jugée lourde au pas horaire, se simplifie au fur et à mesure que la durée étudiée est plus longue. Des chroniques continues de pluies peuvent être simulées. Associées à une modélisation de la pluie en débit, elles génèrent des chroniques continues de débit, disponibles pour prédéterminer les situations de crise, étudier les règles de gestion d'un ouvrage de stockage... Remarquons aussi, que le passage par un modèle de transformation de la pluie en débit, offre la possibilité de simuler l'impact d'activités anthropiques : reforestation, urbanisation, incendie de forêt, évolution climatique... Certes les marges d'incertitude sont encore trop fortes, mais des éléments de réponse peuvent être apportés contrairement à une stricte approche statistique.

\section{III — L'ESTIMATION RÉGIONALE DE LA RESSOURCE EN EAU}

\subsection{Cadre de l'étude}

La Loi sur l'Eau de 1992 pose les bases d'une gestion concertée de l'eau : SDAGE, SAGE, débits de référence d'étiage... La norme pour l'instruction des dossiers d'autorisation ou de déclaration des rejets et des prélèvements en rivière (décret 93-742 de mars 1993) est le débit mensuel d'étiage de fréquence quinquennale sèche (QMNA5). Les débits réservés des cours d'eau à l'aval des aménagements s'expriment comme un pourcentage du débit annuel moyen ou module (décret $84-512$ de juin 1984). Les dispositions prises par le législateur posent toutefois le problème de la connaissance de ces débits de référence en tout point du réseau hydrographique du territoire. Le débit de quelques cours d'eau est certes connu au droit des stations de jaugeage. Mais I'hydraulicité reste une inconnue pour la majorité d'entre eux. Confrontées à ce problème, les DIREN des régions Corse, Languedoc-Roussillon et Provence-AlpesCôte d'Azur ont souhaité disposer d'une méthode d'estimation régionale.

\subsection{Les traitements des données pluviométriques et débitmétriques}

\subsubsection{La zone d'étude et les données}

La zone d'étude actuelle concerne trois régions : Languedoc-Roussillon, Corse et Provence-Alpes-Côte d'Azur, soit 13 départements (50 $000 \mathrm{~km}^{2}$ environ). La climatologie de cette zone est extrêmement contrastée : du climat méditerranéen côtier au climat de haute montagne. La pluviométrie annuelle moyenne varie dans une fourchette de 500 à $2000 \mathrm{~mm}$, ce qui induit une variabilité hydrologique marquée :

- le module est de l'ordre de $5 \mathrm{l} / \mathrm{s} / \mathrm{km}^{2}$ près de la côte ; il atteint plus de $70 \mathrm{l} / \mathrm{s} / \mathrm{km}^{2}$ pour les bassins de montagne.

- le QMNA5 varie de 0 à plus de $10 \mathrm{l} / \mathrm{s} / \mathrm{km}^{2}$.

La quasi-totalité de l'information hydrologique existante a été traitée : 276 bassins versants ( $94 \%$ ont une superficie inférieure à $1000 \mathrm{~km}^{2}$ et pour $44 \%$ d'entre eux la surface est inférieure à $100 \mathrm{~km}^{2}$ ), environ 600 stations pluviométriques et une quinzaine de points de mesure de l'évapotranspiration potentielle.

\subsubsection{Le calage du modèle pluie-débit}

Le modèle retenu (GR2M) fonctionne au pas de temps mensuel. Ce modèle, de type conceptuel, est réglé par deux paramètres $[1,2]$. Les valeurs de ces deux paramètres sont déduites d'une période d'apprentissage durant laquelle sont comparés les débits observés et ceux calculés par le modèle grâce à la seule connaissance des pluies de chaque mois. A travers ces valeurs, on vise bien sûr à réduire l'écart entre débits observés et débits calculés.

\subsection{Performances de la modélisation}

L'ensemble de l'information a été traité par le logiciel LOIEAU. Pour chacun des bassins versants pour lesquels 


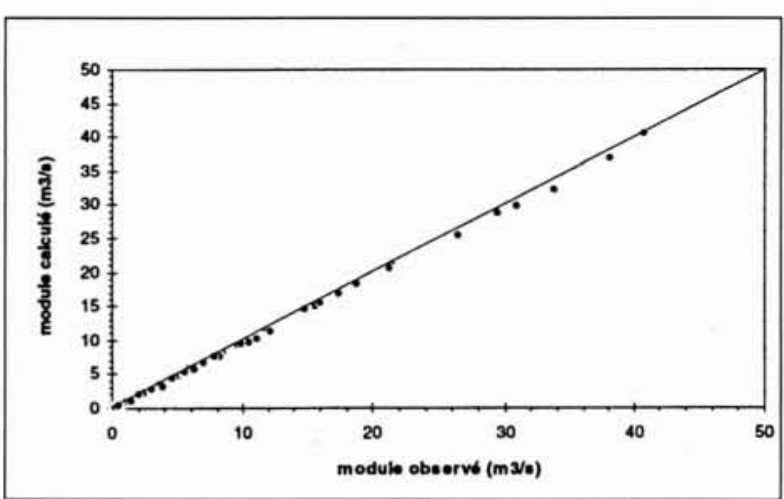

2. Comparaison des modules calculés sur les séries observées et sur les séries calculées par le modèle (période de calage). Exemple de la région LanguedocRoussillon.

une chronique de débits était disponible, le logiciel a assuré, à partir des données pluviométriques locales, le calcul des pluies mensuelles du bassin et leur transformation en débit par GR2M. Bassin par bassin, le logiciel a optimisé les valeurs des deux paramètres afin de reproduire au mieux les débits observés. Les figures 2 et 3 comparent les modules et débits mensuels d'étiage de fréquence quinquennale de la chronique observée à ceux de la chronique simulée par le modèle (pour la région Languedoc-Roussillon). Ce sont, en fait, les performances du modèle qui sont présentées quant à sa capacité de restituer correctement les débits de référence.

Les modules sont très bien reproduits ; le coefficient de détermination du nuage de points (valeur observée, valeur simulée) est pratiquement égal à l'unité. Bien que des écarts soient notables sur les QMNA5, le modèle n'entraîne pas de biais systématique ; le coefficient de détermination du nuage de points (QMNA5 observé, QMNA5 simulé), atteint 0,91.

\subsection{Régionalisation des paramètres du modèle et per-} formances

Le report cartographique (non reproduit ici [3|) des deux paramètres du modèle pluie-débit montre une certaine organisation spatiale, qui fait nettement apparaître l'influence des régimes hydrologiques. C'est un excellent résultat dans la mesure où l'on remarque qu'un modèle paramétré par seulement deux coefficients permet de simuler des chroniques continues de débits mensuels.

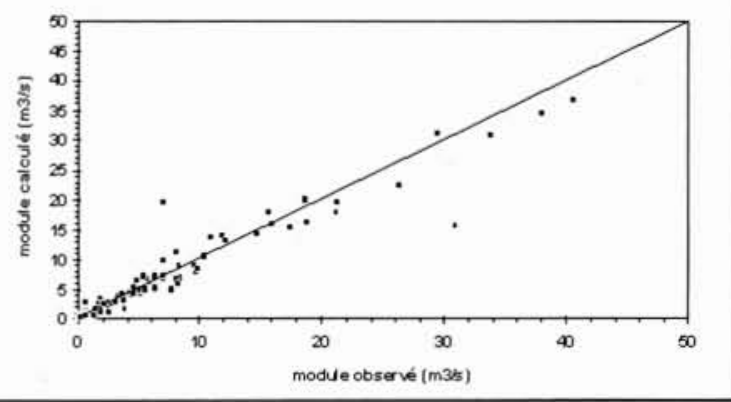

4. Comparaison des modules calculés sur les séries observées et sur les séries calculées par le modèle régionalisé (valeurs régionalisées). Exemple de la région Languedoc-Roussillon.

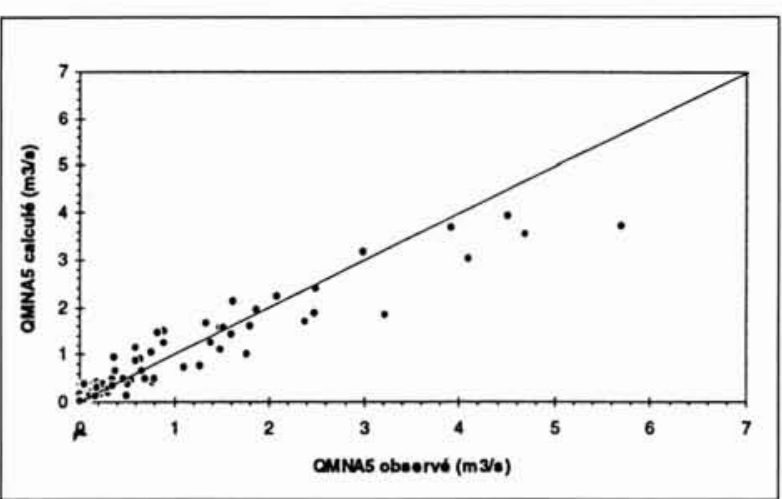

3. Comparaison des débits d'étiage de fréquence quinquennale sur les séries observées et sur les séries calculées par le modèle (période de calage). Exemple de la région Languedoc-Roussillon

\subsubsection{Performances du modèle après régionalisation}

Comme précédemment, nous comparons, figures 4 et 5 , les modules et QMNA5 des séries observées et des chroniques simulées par le modèle utilisé avec les paramètres régionaux. Les résultats se dégradent mais restent très corrects :

- explication de $95 \%$ de la variance des modules ;

- le pourcentage d'explication est de $75 \%$ pour les QMNA5.

\subsection{Le logiciel LOIEAU}

\subsection{Présentation du logiciel}

Le traitement de l'information pluviométrique et hydrométrique disponible a permis de dégager une méthodologie opérationnelle de génération de chroniques de débits mensuels. De plus, la régionalisation de la méthode permet son utilisation sur l'ensemble de la zone d'étude. L'utilisation de cette méthode a impliqué toutefois le développement d'un outil informatique pour effectuer l'ensemble des opérations nécessaires au calcul. Cela est assuré par le logiciel LOIEAU dont l'écriture sous Windows offre une excellente convivialité à l'utilisateur [2].

A partir des bases de données cartographiques, climatologiques et hydrologiques incorporées au logiciel, LOIEAU propose une démarche automatique, tout en laissant à l'utilisateur la possibilité d'intervenir à chaque étape de calcul :

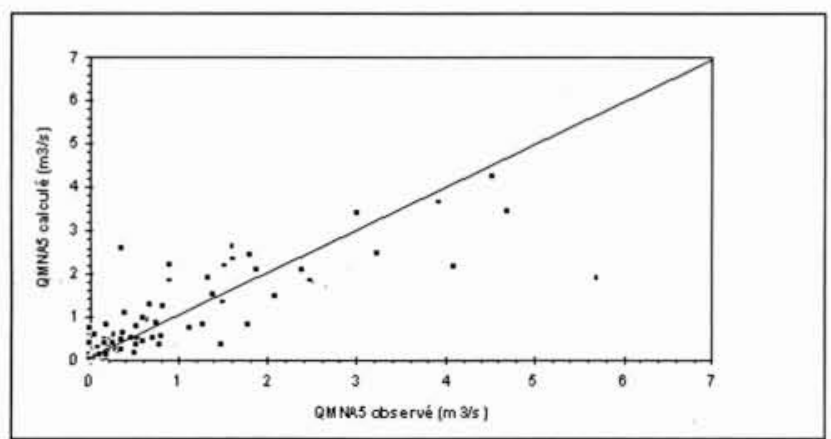

5. Comparaison des débits d'étiage de fréquence quinquennale sur les séries observées et sur les séries calculées par le modèle régionalisé (valeurs régionalisées). Exemple de la région Languedoc-Roussillon. 
- les limites d'un bassin versant quelconque peuvent être tracées à l'écran à l'aide de la souris ou lues dans un fichier :

- calcul de la surface et de l'évaporation mensuelle moyenne ;

- choix d'un poste pluviométrique, ou intégration automatique pour estimer la pluviométrie mensuelle durant la période de génération choisie par l'utilisateur ;

- possibilité d'intervenir sur le paramétrage automatique du modèle pluie-débit, proposé par LOIEAU ;

- génération d'une chronique mensuelle de débits ;

- outre la restitution du fichier des débits mensuels, LOIEAU effectue les statistiques de l'ensemble des variables afin d'estimer le module et le débit mensuel d'étiage de fréquence quinquennale ;

- pour un site jaugé, LOIEAU propose un contrôle des performances de la modélisation par comparaison graphique.

\subsubsection{Utilisation}

Outre l'estimation des débits de référence, LOIEAU offre d'autres possibilités :

- pour un bassin versant dont les écoulements sont mesurés, LOIEAU permet :

- la reconstitution des données manquantes,

- l'extension des chroniques de débits mensuels,

- l'examen de la représentativité du bassin versant dans son environnement régional grâce à l'examen des paramètres du modèle,

- la possibilité d'utilisation en prévision de débits mensuels après avoir contrôlé les performances lors des années précédentes...

- en tout point du réseau hydrographique, l'utilisateur dispose d'une chronique de débits mensuels utilisable :

- pour toute confrontation ressource-besoin,

- pour l'étude de la faisabilité d'une retenue d'eau,

- pour appréhender les variations saisonnières et interannuelles de la ressource en eau dans le cadre de l'élaboration d'un SAGE...

\subsubsection{Evolution à court terme}

Deux niveaux d'évolution peuvent être distingués : l'amélioration des performances du logiciel, en étroite liaison avec la disponibilité de banques de données géographiques, et l'extension géographique de la zone d'application.

\section{SHYPRE : SIMULATION D'HYDRO- GRAMMES POUR LA PRÉDÉTERMINA- TION DES CRUES}

\section{- 4.1. Présentation de la méthode}

SHYPRE est conçue pour travailler aux pas de temps journalier et horaire. Les deux modèles sont connectables, ce qui offre la possibilité, par exemple, de traiter le problème de gestion d'une retenue en continu au pas journalier, et d'aborder le fonctionnement en crue de la retenue à faible pas de temps. SHYPRE associe une génération stochastique des pluies aux pas journalier et horaire et une modélisation déterministe de la pluie en débit. Ainsi, outre la résolution des problèmes de connaissance et de gestion de la ressource en eau superficielle, la méthode permet d'aborder, par géné- ration de scénarios hydrologiques, la distribution temporelle des crues et leur distribution de fréquence (du courant à l'exceptionnel).

\subsection{La modélisation stochastique des pluies}

Les développements actuels des recherches sur la thermodynamique de l'atmosphère et les processus de génération des pluies ne permettent pas encore d'envisager une modélisation physique opérationnelle. L'ingénierie fait appel aux statistiques et aux probabilités, pour construire des modèles stochastiques de génération. Ces modèles sont généralement articulés en trois phases :

i) identification des variables structurantes de la pluie, indépendantes les unes des autres,

ii) identification des lois de probabilité qui représentent au mieux leur distribution de fréquence,

iii) simulation de longues chroniques par construction à partir des variables structurantes générées.

Un simulateur de nombres aléatoires génère des nombres dans l'intervalle $] 01$ 1| qui sont identifiés à une probabilité ; I'introduction de cette probabilité dans la loi de probabilité inverse permet alors de générer une réalisation de la variable.

De nombreux auteurs proposent des structures de génération des pluies journalières. Nous avons retenu un modèle à cinq variables : durées sèches, durées humides, hauteurs de pluie journalière selon que le jour pluvieux est isolé ou situé au centre ou aux extrémités de la séquence pluvieuse. Le calage d'un tel modèle à partir d'une chronique d'observation de pluies journalières ne pose pas de problème particulier.

La construction d'un modèle de génération de pluies horaires est un plus délicate. F. Cernesson [4], et P. Arnaud [5] ont développé un modèle à huit variables : le nombre d'épisodes pluvieux par an (un épisode pluvieux comporte un total journalier qui excède $20 \mathrm{~mm}$ ), le nombre de périodes pluvieuses par épisode, la durée sèche entre deux périodes pluvieuses, le nombre d'averses par période, la durée et l'intensité des averses, le rapport entre l'intensité horaire maximale et l'intensité de l'averse et sa position dans l'averse.

L'étude d'une cinquantaine de postes pluviographiques du pourtour méditerranéen français a permis de déterminer les lois de probabilité théoriques qui représentent au mieux les distributions de fréquence des différentes variables descriptives du modèle. Pour le calage des lois les plus sensibles à l'échantillonnage, les échantillons ont été préalablement agglomérés après une transformation adimensionnelle des variables initiales.

Le modèle génère des hyétogrammes de pluie horaire, desquels sont extraites les pluies maximales de différentes durées pour chacun des événements simulés. On approche ainsi, de manière empirique, les distributions de fréquence des pluies. On notera, figure 6 , la bonne adéquation du modèle aux échantillons de pluie de durée 1, 3 et 24 heures, pourtant non pris en compte directement par la modélisation, et le comportement surexponentiel des distributions de fréquence. Ce comportement résulte de la structuration des averses au sein d'un épisode pluvieux (phénomène de persistance), alors que la loi des averses, calée sur un très grand échantillon (10 000 valeurs), est strictement exponentielle. Bien sûr, il y a quelque part une limite physique, liée à la thermodynamique, que le modèle ne prend pas en compte. 


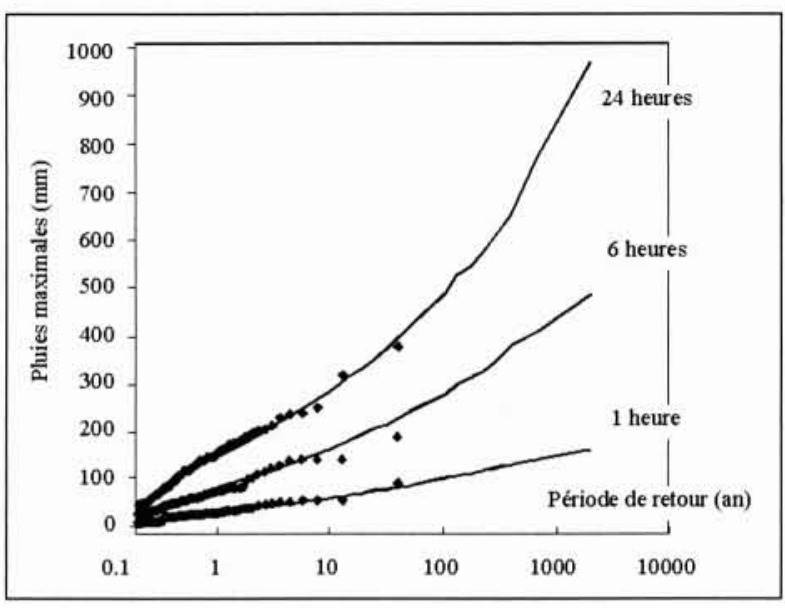

6. Poste pluviographique de Felce (Haute-Corse). Distribution de fréquence des pluies de 1,6 et 24 heures obtenues par simulation (les points représentent les observations).

\subsection{La modélisation de la pluie en débit}

La vocation opérationnelle de la méthode a conduit notre choix vers des modèles simples et robustes, de transformation de la pluie en débit. Bien que la précision des résultats soit importante, elle ne doit pas inciter à une surparamétrisation des modèles qui serait préjudiciable à un transfert des informations vers des bassins versants non jaugés. Il est clair que l'objectif poursuivi vise le développement d'une méthode applicable pour des bassins versants jaugés ou non. La méthode, notamment pour le calage des modèles de crue, se heurte à la parcimonie de l'information pluie-débit à faible pas de temps. Cela peut être partiellement résolu par le transfert d'information journalière vers la modélisation des débits de crue, ce qui implique toutefois une certaine analogie de conception du modèle journalier et du modèle horaire.

\subsubsection{Les modèles GR}

La structure des modèles GR [6] est identique au pas journalier et au pas horaire. Dans leur version à trois paramètres, ces modèles de type à réservoir, impliquent le calage d'un paramètre de production et deux paramètres de transfert.

Le modèle au pas journalier a été largement utilisé (sur 300 bassins versants environ). Arnaud [5] a étudié, à partir d'un calage sur 17 bassins versants de la zone méditerranéenne française, la possibilité de couplage des versions journalière et horaire. Cette confrontation aboutit à des résultats intéressants :

i) les performances du modèle horaire ne sont pas altérées si on retient le même paramètre de la fonction de production journalière et horaire,

ii) le taux de remplissage du réservoir qui règle la production journalière est un bon descripteur de l'état hydrique du bassin. Cette information peut être utilisée lors du couplage des deux pas de temps. Il est à remarquer que cette information est extrêmement intéressante car tous les modèles de crue se heurtent à l'initialisation des réservoirs. On entrevoit ici une application intéressante pour les modèles de prévision de crue, qui pourraient être avantageusement initialisés par l'information d'un modèle journalier, iii) le modèle horaire, en utilisation pour la prédétermination des débits, n'est que peu sensible à un des paramètres de transfert (le temps de montée de l'hydrogramme unitaire). Dans la conception du modèle, ce paramètre reproduit le décalage temporel entre la pluie et les écoulements. Il n'est pas étonnant que les performances intemporelles (en prédétermination) du modèle ne soient pas altérées,

iiii) des recherches supplémentaires restent encore nécessaires pour prédéterminer le dernier paramètre en l'absence d'information horaire.

\subsubsection{Modélisation dérivée de la théorie de l'hydro- gramme unitaire}

L'association d'une fonction de production simple (coefficient d'écoulement, fonction du Soil Conservation Service (SCS), modèle d'infiltration de Horton...) à un hydrogramme unitaire est largement utilisée pour construire des hydrogrammes de projet. La simplicité de la méthode se heurte toutefois à la définition d'un temps caractéristique, dont le choix influence sensiblement les résultats. Dans notre approche, la disponibilité de formes multiples de hyétogrammes évacue en partie ce problème. D'autre part, comme en témoigne la figure 7 , ces méthodes simples ne reproduisent que très approximativement la réalité.

Il apparaît que ces faibles performances sont en grande partie liées à l'hypothèse de linéarité de l'hydrogramme unitaire. On peut pallier à ceci, sans complication majeure, en introduisant de la non-linéarité. Nos recherches nous ont conduit à modifier automatiquement la forme de l'hydrogramme unitaire en fonction de l'intensité de la pluie. On aboutit ainsi à un modèle simple à seulement deux paramètres dont les performances se rapprochent du modèle GR3H à trois paramètres (figure 7).

Les avantages de cette modélisation se situent dans l'approche simplifiée des paramètres. L'utilisation de la fonction de production du SCS permet une détermination immédiate du paramètre de production du modèle de crue grâce à la seule information journalière. Et des critères physiques simples : surface du bassin versant, longueur du plus long axe drainant, pente des drains..., facilement accessibles avec un modèle numérique de terrain, permettent d'approcher le paramètre de l'hydrogramme unitaire.

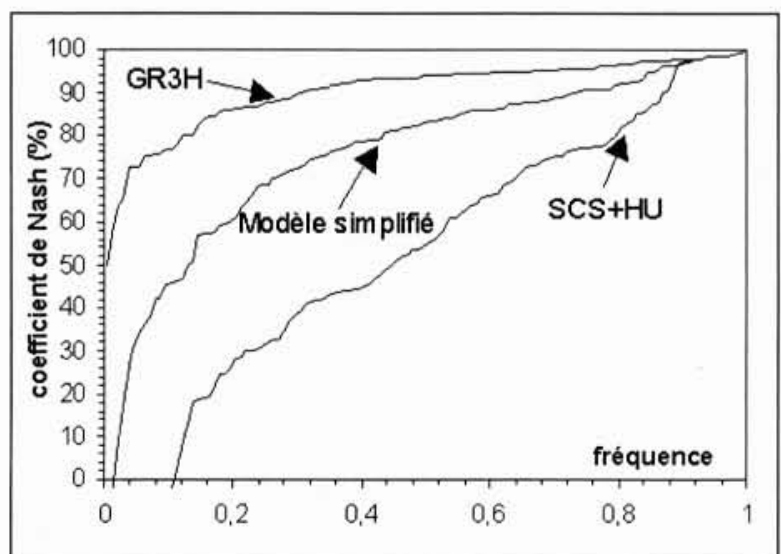

7. Bassin versant du Rimbaud. Distribution des coefficients de Nash, obtenus par calage événementiel des modèles GR3H, SCS+HU, et le modèle modifié à deux paramètres. 


\subsection{Les diverses utilisations de la méthode}

La gestion journalière de la ressource en eau superficielle peut être avantageusement abordée grâce aux chroniques de débits simulés sur de très longues durées (pas de limite de calcul). L'impact de multiples scénarios de gestion peut ainsi être apprécié. Il est clair que la disponibilité de chroniques d'informations facilite la mise en œuvre. En l'absence d'information, la méthode est applicable par transfert d'information des sites jaugés voisins. On ne peut que redouter, sans pouvoir réellement l'apprécier, une marge d'incertitude plus grande.

Pour la connaissance des débits de crue, la méthode génère de multiples scénarios d'hydrogrammes. Cette procédure évite la construction d'hydrogrammes synthétiques de projet, plus ou moins représentatifs de la réalité et la controverse de leur calage en fréquence. La méthode propose aussi une nouvelle approche de la distribution de fréquence des débits (débits instantanés, débits moyens sur différentes durées, débits seuils...) qui est utilisable pour le calcul des débits sur l'ensemble de la plage de fréquence : du courant à l'exceptionnel. Le transfert d'information journalière vers les modèles horaires permet de pallier, en partie, la faible disponibilité de chroniques pluie-débit à faible pas de temps. Les systèmes d'information géographique (géologie, occupation de l'espace, modèle numérique de terrain) sont un support intéressant de régionalisation de la méthode, qui est actuellement mise en œuvre pour l'étude de synthèse des débits de crue de la Corse.

\section{$\mathrm{V} \square$ CONCLUSION}

Affirmer que les pluies génèrent les écoulements des bassins versants ne représente pas une avancée hydrologique notable. Les techniques hydrologiques courantes n'ont pourtant qu'une utilisation limitée et restrictive de l'information pluviométrique. La puissance des moyens de calcul, la disponibilité d'information géographique, ouvrent de nouvelles perspectives à la modélisation de la pluie en débit.

La note présente différents outils (LOIEAU, SHYPRE) qui permettent d'aborder les problèmes de la disponibilité régionale de la ressource en eaux superficielles, de la gestion des retenues d'eau au pas journalier, de la prédétermination des débits de crue par simulation de scénarios d'hydrogrammes. L'approche régionale (menée sur la façade méditerranéenne française), garantit une robustesse certaine à ces approches. L'intérêt méthodologique de ces méthodes se situe dans des modélisations moins sensibles à l'échantillonnage que de simples statistiques et susceptibles de prendre en compte l'impact des activités anthropiques. En outre, ces techniques de simulation proposent une approche originale pour la prédétermination des débits de crue sur l'ensemble de la plage des fréquences.
Malgré leur rusticité (et cela est aussi certainement un avantage), ces méthodes ont un avenir certain en relation avec les systèmes d'information géographique, pour développer des outils conviviaux utilisables par l'ingénierie.

\section{REMERCIEMENTS}

Le transfert des résultats de recherches dans des applications opérationnelles a été possible grâce à l'initiative des DIREN des régions Corse, Languedoc-Roussillon et Provence-Alpes-Côte d'Azur. Outre le soutien financier, leur implication a été permanente tout au long du développement des études. Nous tenons à remercier particulièrement $\mathrm{Ch}$. Pasquier, Cl. Millo, R. Claudet, G. Le Gac et Cl. Benech pour leur étroite collaboration.

\section{BIBLIOGRAPHIE}

II MAKHLOUF Z.. MICHEL. Cl. (1994). A two-parameter monthly water balance model for French watersheds. Journal of Hydrology 162, p. 299 318.

12] LAVABRE J., CAMBON J.P., FOLTON N., MAKHLOUF Z., MICHEL Cl. (1997). LOIEAU : un logiciel pour l'estimation régionale de la ressource en eau. Application à la détermination des débits de référence de la région méditerranéenne française. In Ingénieries EAT, $n^{\circ} 12$ décembre 1997, pp. 59-66.

[3] CEMAGREF, DIREN (1996). Etude des débits de référence des régions Languedoc-Roussillon, Corse et Provence-Alpes-Côte d'Azur. Note de synthèse et présentation du logiciel LOIEAU. Note interne, 56 pages.

[4] CERNESSON F., (1993). Modèle simple de prédétermination des crues de fréquences courante à rare sur petits bassins versants méditerranéens Thèse de doctorat de l'Université Montpellier II, 240 p + annexes.

[5] ARNAUD P. (1997). Modèle de prédétermination de crues basé sur la simulation. Extension de sa zone de validité, paramétrisation du modèle horaire par l'information journalière et couplage des deux pas de temps. Université Montpellier II. Diplôme de Doctorat (21/11/97). 258 p. + annexes.

[6] MICHEL Cl., EDIJATNO, LEVIANDIER T. (1991). Progrès et application de la modélisation conceptuelle pluie-débit. Rapport quadriennal 1987-1990 pour la XXème Assemblée Générale de I'Union Géodésique et Géophysique Internationale à Vienne, 11-24 août 1991. Publié avec le concours de l'Académie des Sciences de Paris, p. 219-222.

[7] ARNAUD P ., LAVABRE J. (1997). Simulation du fonctionnement hydrologique d'un bassin versant : application à la conception et à la gestion d'un barrage. In Ingénieries EAT. ${ }^{\circ} 120$, juin 1997. p. 43-54.

[8] ARNAUD P., PICARD S., LAVABRE J., DOUGUEDROIT A. (1997). Modélisation stochastique des pluies horaires. Application à la région méditerranéenne française. Association Internationale de Climatologie, 10ê colloque international de climatologie, Québec, 9-12 sept. 1997. 10 p.

[9] MAKHLOUF Z . (1994) Compléments sur le modèle pluie-débit GR4J et essai d'estimation de ses paramètres. Thèse de l'université Paris XI Orsay.

[10] USDA-SCS (1985). National Engineering handbook, section hydro$\log y$. 